\title{
Valores econômicos para habilidade de permanência e probabilidade de prenhez aos 14 meses em bovinos de corte
}

\author{
[Economic value for stayability and heifer pregnancy at 14 months in beef cattle herds]
}

\author{
I.B. Formigoni, J.B.S. Ferraz, J.A.II.V. Silva, J.P. Eler, R.C. Brumatti
}

Faculdade de Zootecnia e Engenharia de Alimentos - Departamento de Ciências Básicas - USP

Av. Duque de Caxias, 225 - Norte - Caixa Postal 23

13635-970 - Pirassununga, SP

\begin{abstract}
RESUMO
Estimaram-se os valores econômicos para as características reprodutivas de probabilidade de prenhez aos 14 meses (PP14) e habilidade de permanência (HP). Foram simulados dados de uma propriedade pecuária de corte, especializada na fase de cria para aplicação de modelagem bioeconômica. A PP14 foi avaliada considerando taxas percentuais crescentes de prenhez de novilhas aos 14 meses de idade, e a HP foi avaliada a partir da diminuição da taxa de descarte de vacas. O valor econômico da PP14 foi estimado em $\mathrm{R} \$ 695,97$ por unidade percentual de prenhez de novilhas aos 14 meses, avaliada para o sistema produtivo, enquanto o valor econômico da HP variou em função do custo de compra da novilha de reposição. Os valores, avaliados por unidade percentual de diminuição na taxa de descarte de vacas, foram de $\mathrm{R} \$-2.140,00, \mathrm{R} \$-140,00$ e $\mathrm{R} \$ 2.860,00$ para o custo de compra da novilha de $\mathrm{R} \$ 500,00, \mathrm{R} \$ 700,00$ e $\mathrm{R} \$ 1.000,00$, respectivamente. Os valores econômicos negativos para HP indicaram que o valor de descarte da vaca superou o custo de compra da novilha. Quanto maior for o valor investido na reposição de novilhas maior será a importância econômica do HP.
\end{abstract}

Palavras-chave: bovino de corte, característica reprodutiva, modelo bioeconômico

\begin{abstract}
Economic values for two reproductive traits, percentage of heifers pregnant at 14 months of age (PP14) and stayability (HP), were estimated using simulated bioeconomic modeling data for a cow-calf production system. Changes in HP were modeled as decreases in percentage of annual culling of cows. The economic value of PP14 was $R \$ 695.97$ per one unit increase in percentage of heifers pregnant at 14 months of age, while the economic value of HP varied according to the simulated cost of replacement heifers. Economic values per unit decrease in cow culling percentage were $R \$-2,140.00, R \$-140.00$ and $R \$ 2,860.00$ for heifer prices of $R \$ 500, R \$ 700$ and $R \$ 1,000$, respectively. Economic values for HP were negative when the salvage value of a cull cow was higher than the cost of a replacement heifer. Thus higher investment for replacement heifers increases the importance of stayability in a cattle herd.
\end{abstract}

Keywords: beef cattle, reproductive trait, bioeconomic model

Projeto financiado pela FAPESP

Recebido para publicação em 1 de setembro de 2003

Recebido para publicação, após modificações, em 29 de setembro de 2004

E-mail: ibformigoni@yahoo.com 


\section{INTRODUÇÃO}

A inclusão de características reprodutivas nos objetivos de seleção é de extrema importância para a pecuária de corte nacional (Pereira, 2000; Eler et al. 2001). Tal fato pode ser comprovado pelos estudos econômicos conduzidos para as condições de produção nacional, que indicam serem as características relacionadas à fertilidade do rebanho como as mais economicamente importantes (Bittencourt, 2001; Brumatti, 2002; Formigoni, 2002). Adicionalmente, Newman et al. (1992), Macneil et al. (1994) e Phocas et al. (1998) relataram a importância econômica das características de fertilidade sobre as demais características de peso e qualidade de carcaça.

Eler et al. (2001) destacaram que o produtor de bovinos de corte busca, basicamente, a precocidade sexual e a obtenção de um bezerro/vaca/ano. Os autores sugerem que a probabilidade de prenhez aos 14 meses (PP14) e a habilidade de permanência (HP) têm-se firmado como critérios de seleção em bovinos de corte no Brasil, especialmente para a raça Nelore.

Segundo Eler et al. (2002), a PP14 é uma opção de seleção de baixo custo, requerendo apenas que novilha próxima aos 14 meses seja exposta ao touro ou inseminada, independente do peso e da condição corporal. A HP refere-se principalmente à vida reprodutiva de uma matriz, indicando sua capacidade em permanecer no rebanho produzindo um bezerro ao ano, sem falhas, até uma idade específica, conforme definição de Snelling et al. (1995).

Short et al. (1990) e Charteris et al. (1999) apresentaram cálculos da importância econômica para PP14 e HP, respectivamente. Contudo, Charteris et al. (1999) destacaram a ausência de estudos econômicos das características inclusas nos programas de seleção, especialmente para as características relacionadas à fertilidade do rebanho. Formigoni et al. (2002) abordaram a necessidade de estimar valores econômicos para características recentemente incorporadas à rotina de seleção dos produtores nacionais, especialmente a PP14 e a HP.
Para estimar os valores econômicos, Bourdon (1998) e Meszaros (1999) recomendaram o uso de modelos bioeconômicos, abordados como ferramentas que permitem a descrição dos aspectos biológicos e econômicos das características e do cenário produtivo do sistema. Bourdon (1998) revelou como principal vantagem do modelo bioeconômico a precisão, representando do modo mais próximo do real o conjunto de operações e as particularidades inerentes à propriedade.

O objetivo deste trabalho foi avaliar os aspectos econômicos da seleção das características reprodutivas PP14 e HP, em rebanhos bovinos de corte no Brasil na fase de cria, de acordo com um cenário produtivo representativo dos sistemas de produção inseridos nos programas de melhoramento genético animal.

\section{MATERIAL E MÉTODOS}

$\mathrm{O}$ estudo seguiu a rotina indicada por Ponzoni e Newman (1989), que caracterizaram o desenvolvimento dos objetivos de seleção seguindo as fases de: (i) especificação do sistema produtivo; (ii) identificação das fontes de rendimentos e despesas no rebanho comercial; (iii) determinação das características biológicas que influenciam os rendimentos e as despesas; e (iv) estimação de valores econômicos para cada característica componente dos objetivos de seleção.

O cenário produtivo simulado foi estabelecido para um sistema de produção em pasto, com animais zebuínos (anelorados), monta natural entre novembro e janeiro (90 dias), nascimentos entre agosto e outubro e desmama de fevereiro a maio. Foi considerada: idade base à primeira cobrição aos 24 meses de idade e recria das novilhas necessárias à reposição anual de matrizes. O restante das fêmeas desmamadas, juntamente com os machos, foram vendidos. Os valores salvos no descarte da vaca e touro foram considerados como fontes de receita para a propriedade simulada. A estrutura do rebanho base simulada para o estudo é apresentada na Fig. 1. 


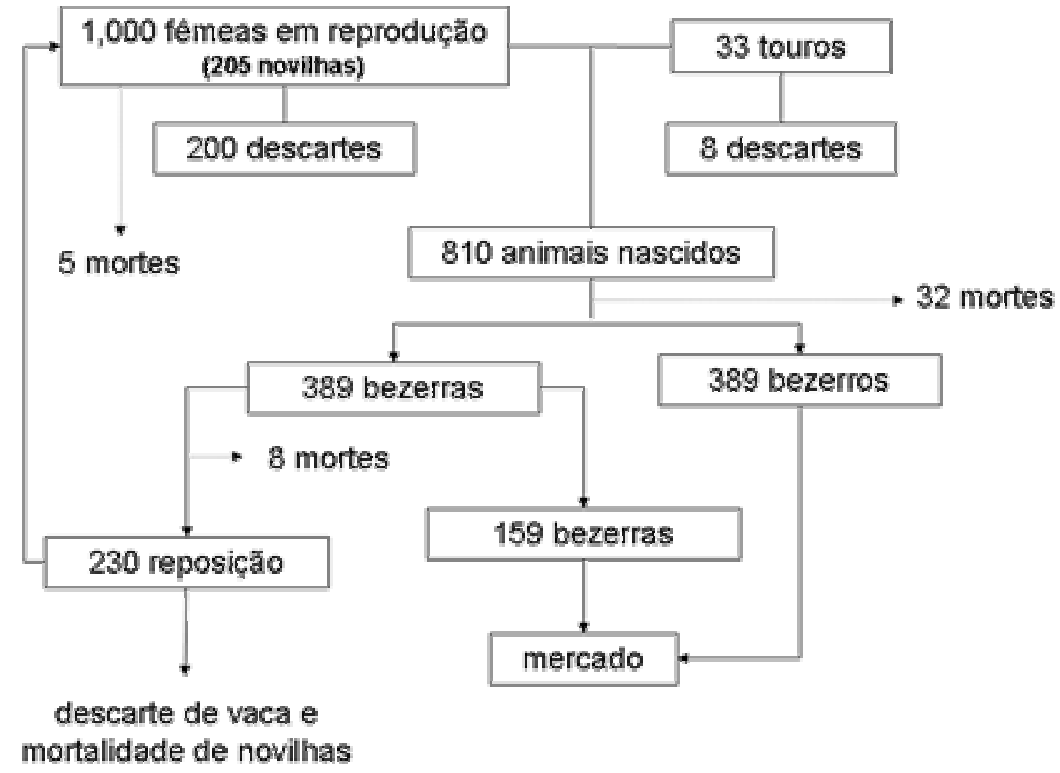

Figura 1. Estrutura e fluxo do rebanho bovino estabilizado, de acordo com a simulação do estudo.

Como a análise retrata o perfil das propriedades que produzem bezerros desmamados para venda e fazem uso do melhoramento genético, os dados de desempenho médio do rebanho caracterizaram sistemas com níveis tecnológicos e produtividade elevados, conforme valores apresentados por Zimmer et al. (1998).

O valor econômico da PP14 foi estimado a partir dos efeitos nos custos, nas receitas e conseqüentemente, no lucro do sistema produtivo, aplicando taxas percentuais crescentes de prenhez sobre as novilhas retidas para reposição. Animais anteriormente prenhes aos 24 meses foram gradativamente substituídos por animais prenhes aos 14 meses, sendo igualmente aplicada uma taxa de reconcepção de $50 \%$ das novilhas que emprenharam aos 14 meses (ou seja, a taxa de prenhez das novilhas prenhes aos 14 meses na estação reprodutiva seguinte). Para o peso médio da progênie à desmama produzida por novilha prenhe aos 14 meses, foi descontado o valor de $15 \mathrm{~kg}$ em relação à média dos animais oriundos de fêmeas que pariram a partir de 36 meses.

As relações de lucro e a PP14 foram avaliadas utilizando o procedimento de modelo bioeconômico, em que o lucro marginal referente à PP14 foi calculado a partir da variação de lucro inerente à respectiva mudança no desempenho médio da característica, mantidas constantes todas as demais variáveis componentes do modelo bioeconômico.

Os procedimentos para análise do valor econômico da HP foram adaptados de Charteris et al. (1999), os quais dividiram a análise de lucro da propriedade para cada categoria animal, como segue:

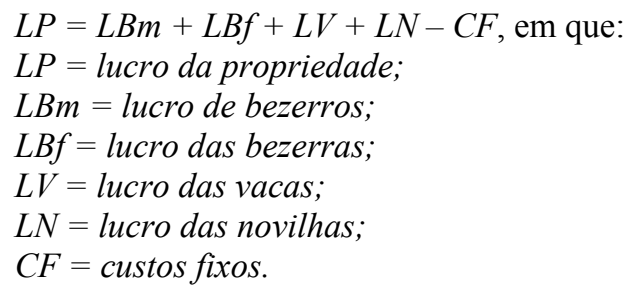

As equações de lucro foram:

$$
\begin{aligned}
& L B m=N \times[(N B D \times P D m \times P m)-(N B D \times \\
& C P m)] \\
& L B f=N \times[(N B D \times P D f \times P f)-(N B D \times C P f)] \\
& L V=N \times[(T D V \times P V \times P v)-C P v] \\
& L N=N \times T D V \times P C n \text {, em que } \\
& N=\text { número de vacas em reprodução; } \\
& N B D=\text { número de bezerros }(\text { as }) \text { desmamados }(\text { as }) ; \\
& P D=\text { peso médio à desmama; } \\
& P=\text { preço de venda ( } m=\text { macho, f=fêmea e }
\end{aligned}
$$


$v=v a c a)$

$C P=$ custos de produção ( $m=$ macho, $f=$ fêmea $e$ $v=$ vaca);

$P V=$ peso médio da vaca;

$T D V=$ taxa de descarte de vaca ao ano;

$P C=$ preço de compra da novilha .

Como os custos fixos não são relevantes para a análise econômica da HP, ou seja, não variam conforme a mudança na característica, foram assumidos como sendo zero. Para estimar os valores econômicos da HP, foram considerados: $\mathrm{N}=1000, \mathrm{NBD}=80 \%, \mathrm{PDm}=175 \mathrm{~kg}, \mathrm{PDf}=165 \mathrm{~kg}$, $\mathrm{PV}=420 \mathrm{~kg}$ e $\mathrm{TDV}=20 \%$.

Para o preço de compra da novilha de reposição, foram considerados os valores de $\mathrm{R} \$ 500,00$, $\mathrm{R} \$ 700,00, \mathrm{R} \$ 1.000,00$ por animal, na tentativa de representar mercados e regiões diferenciadas da pecuária de corte nacional.

Os custos anuais de manutenção foram simulados e baseados na análise bioeconômica desenvolvida, sendo de $\mathrm{R} \$ 74,14$ e $\mathrm{R} \$ 69,90$ por bezerro e bezerra à desmama, respectivamente, e de $\mathrm{R} \$ 177,94$ por vaca.

A importância econômica da HP foi representada pelo lucro marginal resultante da diminuição gradual do percentual da taxa de descarte das vacas, mantendo constante a taxa de fertilidade, como sugerido por Charteris et al. (1999).

As receitas e os custos médios de produção, aplicáveis às condições de produção do estado de São Paulo, foram simulados a partir de dados de custos médios no ano de 2003, julgados relevantes e fornecidos pela Scot Consultoria SA. ${ }^{1}$ Para as receitas, foram considerados valores de $\mathrm{R} \$ 1,92$ e $\mathrm{R} \$ 1,71 / \mathrm{kg}$ para boi e vaca gorda, referentes ao preço médio do mercado pecuário em São Paulo, de novembro de 2002 a novembro de 2003; para bezerros e bezerras à desmama, o valor aplicado foi de $\mathrm{R} \$ 2,30$ e $\mathrm{R} \$ 1,91 / \mathrm{kg}$ de peso vivo, respectivamente.

\section{RESULTADOS E DISCUSSÃO}

Os custos de produção simulados são resumidos na Tab. 1.

\footnotetext{
${ }^{1}$ Scot Consultoria SA. (www.scotconsultoria.com.br)
}

Tabela 1. Custo de produção anual estimado de acordo com o centro de custo

\begin{tabular}{lcc}
\hline \multicolumn{3}{c}{ Custos de produção } \\
\hline Centro de custo & Total anual (R\$) & $\%$ \\
\hline Mão-de-obra & $109.094,40$ & 39,03 \\
Estrutura e equipamentos & $44.055,00$ & 15,76 \\
Pastagem & $23.375,00$ & 8,36 \\
Combustíveis e lubrificantes & $20.000,00$ & 7,15 \\
Impostos & $18.987,20$ & 6,79 \\
Suplementação nutricional & $18.732,20$ & 6,70 \\
Reprodução & $15.937,50$ & 5,70 \\
Custo administrativo & $15.000,00$ & 5,37 \\
Custo veterinário e medicamento & $14.365,10$ & 5,14 \\
Total & $297.546,40$ & 100,00 \\
\hline
\end{tabular}

Os custos de produção simulados no presente estudo demonstram estar, percentualmente, próximos aos obtidos em análise de propriedades de cria disponível em literatura, de acordo com Anuário... (2000). O resultado da análise econômica foi representado pelo lucro anual, $\mathrm{R} \$ 77.386,81$, e pelo lucro por área de pastagem, $\mathrm{R} \$ 91,01$ por hectare ao ano.

Os valores econômicos estimados referiram-se aos custos variáveis da propriedade, uma vez que as despesas fixas, como depreciação e administração, não variaram com a mudança no desempenho das características analisadas.

Com o acréscimo de animais prenhes aos 14 meses e a conseqüente diminuição da categoria de novilhas de 24 meses, os custos de produção gradativamente decresceram, principalmente em decorrência dos custos alimentares e sanitários. A estratégia de expor as novilhas mais precocemente à reprodução cria um excedente de fêmeas em reprodução, o qual foi contabilizado nos custos. Contudo, tal excedente é gradativamente diluído com o acréscimo de percentual positivo de prenhez aos 14 meses.

Certamente, outros custos adicionais podem advir da estratégia de expor mais precocemente as novilhas à reprodução. Como exemplo, podem-se citar custos de infra-estrutura, como a construção de cercas para piquetes diferenciados e de mão-de-obra adicional, entre outros. Esses custos, por não serem diretamente relacionados à característica e serem dependentes das condições de produção de cada propriedade, foram assumidos como arbitrários e não contabilizados na análise econômica da PP14. 
A prenhez positiva das novilhas aos 14 meses incide no aumento da receita, uma vez que o número de animais produzidos na desmama é maior se comparado à estratégia de expor as novilhas aos 24 meses de idade. Os benefícios econômicos da PP14 na receita do sistema produtivo podem ser expressos pelo maior giro de capital e pela possibilidade de uma cria adicional na vida reprodutiva da matriz.

Estabelecida a idade de exposição da novilha à cobrição de 14 meses, para cada unidade percentual de variação positiva na característica, obtiveram-se redução média no custo de produção de $\mathrm{R} \$ 57,60$ e receita média extra de $\mathrm{R} \$ 638,27$ ao ano. O valor econômico da PP14 para o cenário produtivo e econômico estabelecido pela simulação foi estimado em $\mathrm{R} \$ 695,87$ por aumento de um ponto percentual da prenhez de novilhas aos 14 meses.

Os valores econômicos da HP, avaliados para diversos custos aplicados de investimentos com a novilha, podem ser visualizados na Tab. 2 .

Tabela 2. Valor econômico para habilidade de permanência (HP), analisado para diversos custos de investimento com as novilhas

\begin{tabular}{lc}
\hline Custo da novilha (R\$) & Valor econômico (R\$) \\
\hline 500,00 & $-2.140,00$ \\
700,00 & $-140,00$ \\
$1.000,00$ & $2.860,00$ \\
\hline
\end{tabular}

Para os custos de compra da novilha de $\mathrm{R} \$ 500,00$ e $\mathrm{R} \$ 700,00$, o lucro proporcionado pela redução da taxa de descarte das vacas foi negativo, uma vez que a receita obtida com o descarte da vaca, de $\mathrm{R} \$ 715,00$, superou o custo inicial da novilha. Tal fato não ocorreu para o custo de compra de $\mathrm{R} \$ 1.000,00$.

Os resultados da Tab. 2 sugerem que os valores econômicos para HP dependem do custo de compra da novilha de reposição. É importante ressaltar, contudo, que melhor desempenho das novilhas, associado a um preço mais elevado de compra, foi desconsiderado.

Os valores econômicos da HP para os diferentes custos das novilhas e a respectiva relação com a característica PP14 são apresentados na Tab. 3.
Tabela 3. Valores econômicos da habilidade de permanência (HP) para os custos de compra de novilhas e respectiva relação com a prenhez aos 14 meses (PP14)

\begin{tabular}{lc}
\hline Custo da novilha $(\mathrm{R} \$)$ & $\mathrm{HP}$ \\
\cline { 2 - 2 } & $\mathrm{PP} 14$ \\
\hline 500,00 & $-3,07$ \\
$1.000,00$ & $-0,20$ \\
\hline
\end{tabular}

A importância econômica da HP comparada à da PP14 variou conforme o custo da novilha de reposição, sendo mais economicamente importante a HP em relação à PP14 quanto maior o custo de aquisição da novilha.

Os valores do custo de compra da novilha de $\mathrm{R} \$ 1.000,00$ (Tab. 3), próximos aos encontrados por Charteris et al. (1999), sugerem que o impacto econômico da HP é 3,27 vezes superior ao da precocidade sexual da novilha. Contudo, esses valores dificultam classificar, por ordem de importância, qual a característica mais economicamente relevante, pois, no cenário de custos e receitas analisados, a HP terá maior ou menor importância com relação à PP14. Entretanto, quanto maior for o investimento com a compra de novilhas, maior será a importância econômica da HP.

Phocas et al. (1998) relataram ser difícil comparar os resultados entre estudos, pois as características diferem consideravelmente conforme as circunstâncias de produção. Groen (1989) definiu circunstância de produção como o conjunto de fatores que caracterizam cada propriedade, os quais podem causar sensíveis diferenças nos custos e nas receitas entre os sistemas produtivos. Dentre as circunstâncias de produção, Groen (1989) cita especificidades para as circunstâncias naturais, sociais e econômicas, o sistema de produção, os requisitos de mercado e o atual nível médio de produtividade do rebanho, os quais diferem consideravelmente de acordo com os sistemas produtivos em análise.

Vale destacar que os valores econômicos avaliados são expressos em termos absolutos, por não serem expressos em termos de variação genético-econômica, conforme destacaram Barwick (1994) e Ponzoni e Gifford (1990). Os autores descreveram que o produto entre o valor econômico e o desvio-padrão genético aditivo 
resultou na variação genético-econômica. Contudo, os objetivos do estudo foram caracterizar a importância da PP14 e HP no lucro de um sistema produtivo simulado e ilustrar a relação das características com os benefícios econômicos esperados a partir da seleção do rebanho.

Adicionalmente, HP e PP14 são denominadas como características economicamente relevantes, conforme definição proposta por Golden e Bourdon (1999). Os autores comentaram a necessidade de as avaliações genéticas disponibilizarem DEPs para características de expressivo impacto no lucro dos rebanhos comerciais, as quais são denominadas características economicamente relevantes. Elas estão diretamente associadas à rentabilidade do sistema produtivo, pela relação com custos específicos ou com o rendimento a partir da venda de um produto. Estudos econômicos similares e que possam servir de comparação com o presente estudo não estão disponíveis em literatura no Brasil.

\section{CONCLUSÕES}

A característica HP apresentou maior importância econômica em termos absolutos comparada à PP14. Os fatores de produção que interferem no valor econômico da HP devem ser igualmente avaliados por meio de modelos bioeconômicos de análise.

\section{REFERÊNCIAS BIBLIOGRÁFICAS}

\author{
ANUÁRIO DA PECUÁRIA BRASILEIRA. São \\ Paulo:Argos, 2000. 392p.
}

BARWICK, S.A. B-Object: a PC-Program to derive economic weights for beef cattle. In: GENETIC PREDICTION WORKSHOP, 4., 1994, Kansas City. Proceedings... Kansas City: Beef Improvement Federation, 1994. 10p.

BITTENCOURT, T.C.C. Estimativa de ponderadores econômicos para características de importância econômica em gado de corte, usando equações de lucro. 2001. 59f. Tese (Doutorado) - Faculdade de Medicina de Ribeirão Preto, Universidade de São Paulo, Ribeirão Preto.
BOURDON, R.M. Shortcomings of current genetic evaluation systems. J. Anim. Sci., v.76, p.2308-2323, 1998 .

BRUMATTI, R.C. Desenvolvimento de um modelo bio-econômico para determinação de ponderadores econômicos utilizados em indices de seleção em gado de corte. 2002. 113f. Dissertação (Mestrado) - Faculdade de Zootecnia e Engenharia de Alimentos, Universidade de São Paulo, Pirassununga, SP.

CHARTERIS, P.L.; AMER, P.R.; GOLDEN, B.L. Economic values for stayability and heifer pregnancy of beef cattle. J. Anim. Sci., v.77, p.101, 1999. Suppl. 1.

ELER, J.P.; FERRAZ, J.B.S.; SILVA, J.A.II.V. et al. Melhoramento genético da precocidade sexual na raça nelore. In: SIMPÓSIO NACIONAL SOBRE PRODUÇÃO E GERENCIAMENTO DA PECUÁRIA DE CORTE, 2., 2001, Belo Horizonte. Anais... Belo Horizonte: UFMG, 2001. p. 124-130.

ELER, J.P.; SILVA, J.A.II.V.; FERRAZ, J.B.S. et al. Genetic evaluation of the probability of pregnancy at 14 months for Nellore heifers. $J$. Anim. Sci., v.80, p.951-954, 2002.

FORMIGONI, I.B. Estimação de valores econômicos para características componentes de indices de seleção em bovinos de corte. 2002. 80f. Dissertação (Mestrado) - Faculdade de Zootecnia e Engenharia de Alimentos, Universidade de São Paulo, Pirassununga, SP.

FORMIGONI, I.B.; SILVA, J.A.II.V.; BRUMATTI, R.C. et al. Economic aspects of stayability as selection criterion in belef cattle industry in Brazil. In: WORLD CONGRESS ON GENETIC APPLIED TO LIVESTOCK PRODUCTION, 7., 2002, Montpellier. Proceedings... Montpellier, 2002. CD-ROM.

GOLDEN, B.L.; BOURDON, R.M. The sire summary of the future. Rev. Bras. Reprod. Anim., v.23, p.78-81, 1999.

GROEN, A.F. Cattle breeding goals and production circunstances. 1989. 167f. Thesis (Doctoral) - Wageningen Agricultural University, Wageningen, The Netherlands.

MACNEIL, M.D.; NEWMAN, S.; ENNS, R.M. et al. Relative economic values for Canadian beef production using specialized sire and dam lines. Can. J. Anim. Sci., v.74, p.411-417, 1994. 
MESZAROS, S.A. Optimising the objectives and design of breeding programs with the use of genetic algorithms. 1999. 205f. Thesis (Doctor)

- University of the New England, Armindale. Disponível em: <http://www-personal.une.edu. au/ ssmeszaro/thesismaster.pdf $>$ Acessado em: 11 set 2001 .

NEWMAN, S.; MORRIS, C.A.; BAKER, R.L. et al. Genetic improvement of beef cattle in New Zealand: breeding objectives. Liv. Prod. Sci., v.32, p.111-130, 1992.

PEREIRA, E. Análise genética de algumas características reprodutivas e de suas relações com desempenho ponderal na raça nelore. 2000. 56f. Tese (Mestrado) - Faculdade de Zootecnia e Engenharia de Alimentos, Universidade de São Paulo, Pirassununga, SP.

PHOCAS, F.; BLOCH, C.; CHAPELLE, P. Developing a breeding objective for a French purebred beef cattle selection programme. Liv. Prod. Sci., v.57, p.49-65, 1998.

PONZONI, R.W.; GIFFORD, D.R. Developing breeding objectives for Australian cashmere goats. J. Anim. Breed. Gen., v.107, p.351-370, 1990.

PONZONI, R.W.; NEWMAN, S. Development breeding objectives for Australian beef cattle production. Anim. Prod., v.49, p.35-47, 1989.

SHORT, R.E.; STAIGMILLER, R.B.; BELLOWS, R.A. et al. Breeding heifers at one year of age: biological and economic considerations. In: ANNUAL FLORIDA BEEF CATTLE SHORT COURSE, 39., 1990, Florida. Proceedings... Florida, 1990. Disponível em: $<$ http://www.animal.ufl.edu/extension/beef/docu ments/SHORT90/SHORTCRS.HTM $>$. Acessado em: 20 de nov. 2003.

SNELLING, W.M.; GOLDEN, B.L.; BOURDON, R.M. Within herd genetic analyses of stayability of beef females. J. Anim. Sci., v.73, p.993-1001, 1995.

ZIMMER, A.H.; EUCLIDES FILHO, K.; EUCLIDES, V.P.B. et al. Considerações sobre indices de produtividade da pecuária de corte em Mato Grosso do Sul. Campo Grande: EMBRAPA-CNPGC, 1998. 53p. 